\title{
DISEÑO DE PIERNA ARTICULADA PARA EL PLANTEAMIENTO DE MEDIOS
ALTERNATIVOS DE TRANSPORTE
}

\author{
Miguel Uribe Becerra \\ Maestro en Diseño Industrial. \\ Profesor del Departamento de Diseño \\ Universidad del Valle. Cali, Colombia \\ angel.uribe@correounivalle.edu.co
}

\section{Resumen:}

Este artículo se presenta como resultado de proyectos de investigación en biomimética y evidencia la necesidad de desarrollar medios alternativos de transporte que favorezcan la movilidad sostenible. Se presentan reflexiones sobre el impacto medioambiental negativo de los actuales medios de transporte basados en la rueda; se procede a documentar el método de análisis de animales llevado a cabo en la investigación y se concluye con la propuesta de un mecanismo biomimético de pierna articulada.

Palabras Clave: Biomimética, Diseño, Locomoción, Simulación, Sustentabilidad, Mecanismo.

\begin{abstract}
:
This article is presented as a result of research projects in biomimicry, and evidence the need to develop alternative means of transportation, that seek to contribute to sustainable mobility. Reflections on the negative environmental impact of current means of transport based on the wheel, it is documenting the method of analysis of animals carried out research and concludes with the proposal of a biomimetic of articulated leg mechanism.
\end{abstract}

Keywords: Biomimicry, design, transport, simulation, sustainability, mechanism. 


\section{INTRODUCCIÓN}

"La dicotomía entre desarrollo y sostenibilidad es falsa, sin planeta, no hay economía que valga”

Al Gore

Moller (2006:204) afirma que ningún medio de transporte está libre de generar impacto ambiental. Incluso los zapatos producen efectos contaminantes tanto en el momento de la extracción, producción y transformación de las materias primas, como por su fricción y los residuos que genera. Con mayor razón, la producción y circulación de todo tipo de vehículos, caracterizados por requerir procesos industriales más complejos y por desplazarse con una mayor velocidad, se presentan como fuente de contaminación.

Los sistemas de locomoción terrestres desarrollados por los humanos en la actualidad basan su funcionamiento principalmente en el principio de giro para el avance de la rueda, mientras que los mamíferos e insectos utilizan principalmente las piernas. Nuestros vehículos necesitan superficies preparadas, (que debido a la dependencia del transporte, resultan ser una fuente importante de daño al medio ambiente natural por la necesidad de intervención directa de la tierra para ser adaptada al uso de los mecanismos de ruedas). Aún así, es destacable que con las grandes intervenciones en los suelos y el enorme gasto económico y ambiental, un gran porcentaje de la superficie terrestre resulta inaccesible para nuestros principales y masivos medios de transporte. Los animales cuya locomoción se lleva a cabo por medio de patas se pueden mover sobre la mayor parte de los terrenos, y -en principio-, las máquinas de patas también deberían ser capaces de hacerlo. Por otra parte, las piernas al crear huellas discretas causan menos daños al terreno natural. La rueda privilegia la facilidad de propulsión pero compromete la capacidad de movilidad frente a obstáculos, mientras la comodidad de transporte o el aislamiento de las irregularidades del terreno favorece la locomoción mediante patas.

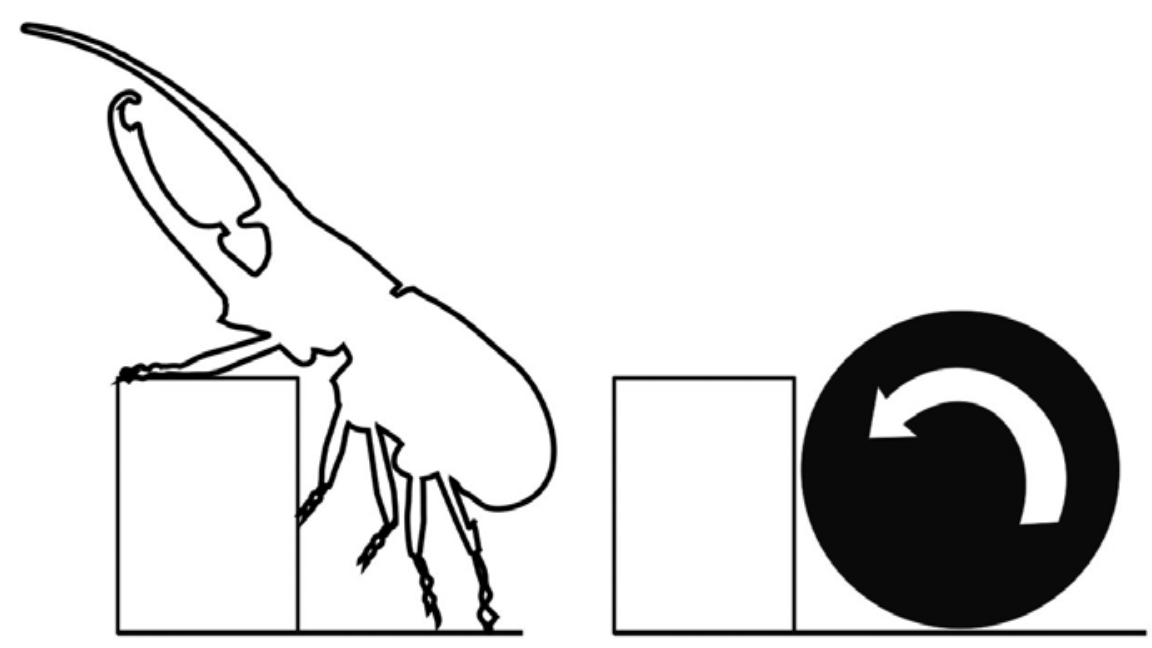

Figura. 1. Los principios biológicos de locomoción en animales proporcionan una gran cantidad de referentes de inspiración para la locomoción terrestre. Los animales que se transportan mediante patas, existen, prosperan y son capaces de superar los obstáculos que están en el orden de su propio tamaño. 
El presente desarrollo teórico avanza en la línea de la elaboración de modelos de representación funcional de mecanismos de locomoción animal terrestre, mediante la comprensión de los principios técnicos y funcionales de las soluciones desarrolladas por la naturaleza que han logrado un alto grado de eficiencia (dado que los animales tienden a desarrollar las mejores estructuras y comportamientos posibles); de esta manera, se intenta dar vía al rompimiento del paradigma del transporte humano con una visión sustentable desde su concepción. El propósito de este artículo es presentar el desarrollo de un modelo de pata articulada como resultado de procesos de investigación y desarrollo biomimético en búsqueda de alternativas de transporte no convencionales y en principio ambientalmente más adecuados.

\section{La necesidad de formas alternativas de transporte}

La sociedad se encuentra actualmente inmersa en dinámicas de consumo y desecho insostenibles; la crisis ambiental es evidente y pone en peligro no solo la prolongación del ser humano como especie, sino también la extinción de muchas formas de vida en el planeta. Es por ello que en diferentes países se han establecido una serie de políticas y lineamientos donde se propone disminuir e incluso revertir las consecuencias adversas causadas por hábitos y conductas ambientalmente inapropiadas. Una de las problemáticas más complejas en el tema ambiental está relacionada con los temas de movilidad: el desarrollo insustentable de los actuales medios de transporte terrestre, donde se necesita no solo una gran cantidad de energía y consumo de recursos en el funcionamiento mismo de los automóviles, sino que se requiere además una inmensa intervención en los espacios, ya que estos deben ser "adaptados" como contextos para el funcionamiento de los automóviles.

El desarrollo de alternativas de mecanismos de locomoción humana se relaciona y justifica en el contexto del fenómeno social de la movilidad. A este respecto se afirma 136 que:

"La disponibilidad de una adecuada infraestructura de transporte urbano, que permita movilizar a personas y bienes de modo digno, oportuno, confiable y económico, integra individualmente aquel núcleo de necesidades básicas comunes. Especialmente relevante para los países en desarrollo - por razones redistributivas (...), se trata de un problema compartido en gran medida por las ciudades del mundo desarrollado: la creciente motorización y extensión del uso del automóvil privado plantea dificultades generalizadas en materia de congestión de tráficos, así como ingentes costos sociales en el campo ambiental y de accidentalidad (por ejemplo a nivel mundial se estima que hacia 2015 las zonas urbanizadas causarán el 80\% de las emisiones de CO2). Ello genera complejos dilemas a las autoridades en el proceso de asignación de prioridades a las inversiones en infraestructura de movilidad urbana". (Lupano Jorge, Sánchez Ricardo, 2009:9). 
Esto plantea un panorama de un estado actual de los dispositivos y las estructuras relativos a la movilidad urbana que se caracteriza por presentar deficiencias con respecto a los requerimientos de las dinámicas y necesidades de desplazamiento contemporáneos alineados con una conciencia ambiental creciente y altamente justificada.

Al analizar algunas de las especies animales se puede determinar que sus sistemas de locomoción no están limitados por los caminos o senderos predispuestos para su funcionamiento, sino que su dotación biológica se acomoda a estos entornos y logran la movilidad de acuerdo con sus necesidades. Los sistemas de locomoción terrestre que actualmente utiliza el hombre están basados principalmente en ruedas, las cuales tienen una relativa eficacia, pero requieren una intervención del medio con el cual interactúan, resultando inviables sin una disposición de una extensa red vial. Es ahí donde el desarrollo de alternativas biomiméticas se presenta como método para emular la integralidad de los sistemas biomecánicos, físicos y energéticos de especies naturales para tomarlas como modelo de equilibrio dinámico con la naturaleza (principal determinante de diseño de artefactos para una locomoción eficiente en diversos entornos). Existe una armonía total entre los desplazamientos de los animales y el entorno natural en el que permanecen, la especialización biológica les ha permitido adaptar su morfología al circum-mundo (su entorno ambiental propio), sus dotaciones biológicas como patas, dientes, pelo, están especialmente adaptados al medio y le sirven para protegerse, funcionar y ser eficientes en el entorno en que viven, sin una afectación que les haya hecho inviable su subsistencia.

A diferencia de los animales, ante las circunstancias, el hombre debe construir un entorno artificial, creando un mundo exterior de objetos o cosas que compensen su inespecialización biológica y que le ayuden a suplir sus necesidades como extensiones de su cuerpo para llevar a cabo tareas que sus limitaciones biológicas le impedirían realizar; el hombre no se adapta al medio (como ocurre con el resto de las especies), sino que adapta el medio como mecanismo de supervivencia.

Estas adaptaciones que el hombre hace de su entorno son, en muchos casos, insustentables y, a pesar de ser poco naturales, parecen corresponder a la misma esencia del hombre: debe enfrentar la naturaleza y no plantear de manera armónica su relación con ella. "Esta reacción contra su contorno, este no resignarse contentándose con lo que el mundo es, es lo específico del hombre. [...] un hombre sin técnica, es decir, sin reacción contra el medio, no es un hombre" (Ortega y Gasset, 1965:24). Los procesos acelerados de producción y consumo incentivados desde la Revolución Industrial, han disparado la producción de desechos, convirtiendo así el tema de los sistemas de transporte en un problema mundial; uno de los factores que incide en la contaminación por uso de automóviles es el crecimiento demográfico, la expansión de las ciudades, además de los cambios en los estándares y estilos de vida, la producción de nuevos productos cada vez más deseables y asequibles para la población. 
Es necesario, desde este punto de vista, generar reflexiones acerca de estrategias alternativas de diseño, orientadas a la búsqueda de soluciones innovadoras para la movilidad o transporte de las personas, entendiendo la movilidad bajo las condiciones de accesibilidad hacia el logro de la subsistencia, el conocimiento y el ocio; bajo principios de sustentabilidad, es decir, bajo la premisa de que ninguna solución será eficiente si, aún cumpliendo su propósito, afecta negativamente la posibilidad de disfrute de los recursos naturales por parte de las generaciones futuras. Es clara la pertinencia del desarrollo de alternativas de mecanismos de locomoción humana menos invasivos con el medio ambiente.

\section{Proceso de diseño apoyado en la Biomimética}

La propuesta de mecanismo de pierna articulada como insumo para el desarrollo de sistemas de transporte alternativos, se origina a partir de la Biomimética, la cual permite la identificación de los principios naturales de locomoción animal terrestre por medio de la investigación y la observación de especies naturales que se presumen "exitosas" en la solución de este problema, de la forma en que las problemáticas de movilidad afectan al sujeto.

La observación cualitativa de los sistemas de locomoción de los animales permite establecer condiciones y variables determinantes que se pueden medir y comparar para evaluar, cuantificar y replicar su efectividad. El fin es utilizar los sistemas de manera innovadora en medios de movilidad para los seres humanos, con la particularidad de que no requieran modificación del contexto en que se utilizan. Janine Benyus, presidenta del Instituto de Biomimética y experta internacional, asegura que el 80 por ciento de las soluciones que buscamos están en el mundo natural. Además, "son ideas que cumplen dos características fundamentales: son soluciones probadas y sostenibles porque han sobrevivido millones de años" .

La Biomimética (bios=vida, mimesis=imitar) se presenta como una disciplina que adopta el uso práctico de mecanismos, funciones, formas y procesos que estudian las ciencias biológicas, para la aplicación en diversas especialidades, entre ellas el Diseño Industrial. Es una ciencia dedicada a imitar el funcionamiento de los seres vivos a través de ingenios mecánicos o robóticos, aplicando diseños naturales para resolver problemas de ingeniería, ciencia de materiales, medicina y otros campos. 
$\mathrm{Si}$ se admite la afirmación de que "un sistema de transporte es eficaz si es sostenible en el tiempo desde parámetros ambientales y sociales" (Miralle Guash y Cebollada, 2003:13), entonces, se pone en evidencia que los actuales sistemas de transporte son, en muchos aspectos, ineficaces y por tanto, difícilmente sostenibles, dado el daño estimado del ambiente y deterioro de la calidad de vida de sus usuarios. En este sentido, se requiere la generación de propuestas tendentes a abordar los aspectos problemáticos y generar mejoras en el estado de los mecanismos y sistemas de transporte, que repercutan de manera positiva sobre las condiciones de movilidad urbana. A este respecto, se afirma que:

"Las exigencias de la movilidad y el diseño de sus posibles soluciones suponen un impacto decisivo sobre la estructura física de las ciudades, y se encuentran por tanto íntimamente vinculadas al planeamiento urbano. La necesidad de reservar para el uso público las vías comunes de movilidad y transporte y su adecuada distribución entre las diversas alternativas modales constituye una parte integral de la planificación del desarrollo urbano". (Lupano Jorge, Sánchez Ricardo, 2009:9).

Los efectos que han operado sobre el ordenamiento y la estructura de las ciudades, los procesos migratorios y de crecimiento recientes (tanto poblacional como en infraestructura) han contribuido a intensificar las demandas de movilidad y transporte de forma dramática. Debido a ello se ha manifestado, cada vez con mayor claridad y urgencia, la necesidad de plantear alternativas desde diversos dominios profesionales (urbanistas, ingenieros, administradores, etc., para proponer soluciones a las problemáticas actuales y los desafíos a mediano y largo plazo de la planeación urbana. En este contexto de constante crecimiento y complejización de los medios urbanos, los diseñadores industriales están llamados a jugar un importante papel en la búsqueda y el desarrollo de opciones de movilidad y transporte, como aportes significativos al mejoramiento de la calidad de vida de los usuarios del sistema de transporte sostenible.

Los términos desarrollo y sostenibilidad fueron popularizados desde el Informe de Brundtland en 1987 y se establecieron como una nueva forma de actuar en la cumbre de Río de Janeiro, en 1992. Allí se definió el Desarrollo Sostenible como: "aquel desarrollo destinado a satisfacer las necesidades de generaciones presentes sin comprometer la capacidad de las generaciones futuras de satisfacer sus propias necesidades”. En cuanto al termino sostenible, una de las posturas más claras de lo que representa es entender los límites físicos y la capacidad finita del planeta de proveer materia, además, lo sostenible se debe tratar más allá de la protección ambiental y debe instaurarse en otros ámbitos, como lo económico, lo social y lo político. Como plantea Bettini "el desafío que supone un desarrollo urbano sostenible, implica la búsqueda de soluciones a los problemas sufridos por las ciudades y a las consecuencias que de ellos se derivan, reconociendo que las ciudades también son capaces de aportar, potencialmente, muchas soluciones a sus propios problemas." (Benetti, 1996:33). 
Ahora bien tres puntos claves de la sostenibilidad urbana según Di pace, son en términos generales el "procurar el bienestar y equidad social, apuntando a la distribución equitativa en el acceso y uso de los recursos, promover la eficiencia económica optimizando la generación de productos y el uso de los recursos naturales y construidos, implicando la minimización de los residuos, así como el uso de tecnologías eficientes y propender la sustentabilidad ecológica entendida como el uso sostenible de los recursos renovables (agua, suelo, aire etc; la minimización del uso de recursos no renovables (petróleo, gas, carbón, etc y el respeto a los limites ecológicos de generación y absorción de residuos" (Di Pace, 2001:29).

En el 2002, en Johannesburgo (Sudáfrica), nuevamente el tema del desarrollo sostenible vuelve a ser debatido, concluyendo que aunque las intenciones han sido buenas, no se han visto buenos resultados en cuanto a los compromisos adquiridos para el desarrollo sostenible con la Agenda 21. En este congreso se ratifica la importancia de entender que para avanzar en una solución para un desarrollo realmente sostenible, es necesario re evaluar los procesos tradicionales de planificación (Velásquez, 2004:3).

Una alternativa de transporte debe concebirse de tal manera que sirva al mejoramiento de la condición tiempo espacio recorrido (Criterio de Eficacia de los Desplazamientos), y como contribución a la solución de los efectos actuales de un conjunto de dimensiones problemáticas de la movilidad urbana (seguridad, accidentalidad, impacto ambiental). De esta manera, la formulación de un proceso de diseño, a través del cual se manifiesta la puesta en práctica de las competencias requeridas para el ejercicio de la profesión de diseñador industrial, se expone en el contexto de una aplicación práctica. La eficiencia es el logro de un objetivo al menor costo unitario posible: esto es, el uso óptimo de los recursos disponibles para alcanzar los objetivos planteados. Esta eficiencia se planteará, en cuanto se puedan establecer las relaciones directas e indirectas de los elementos que constituyen la marcha y que desde lo cualitativo y cuantitativo sean susceptibles de medir, comparar, combinar y establecer posibles ventajas mecánicas. Se establece desde una perspectiva general, la correcta relación entre los tres elementos constitutivos de las extremidades al momento de la locomoción, entrada del movimiento o señal de excitación.

\section{Principios técnicos de las patas animales}

La simulación de diferentes mecanismos de locomoción animal terrestre ha permitido el acercamiento a la comprensión de los principios técnicos y funcionales de estas soluciones, desarrolladas por la naturaleza, logrando un alto grado de eficacia. Apoyados en estos principios se pretende exponer soluciones para el transporte humano, que se adapten a exigencias diversas de manera especializada, intentando dar vía al rompimiento del paradigma del transporte apoyado en vehículos de ruedas como única forma de asistencia posible. 
El proceso de Diseño de la pierna o pata articulada inicia con el análisis de un grupo de animales; se hace una observación con fines de análisis de los segmentos, articulaciones y movimientos que integran el elemento natural. Mediante este proceso de observación directa sobre los especímenes se caracteriza su funcionamiento y se gráfica para representar los componentes y sus relaciones.

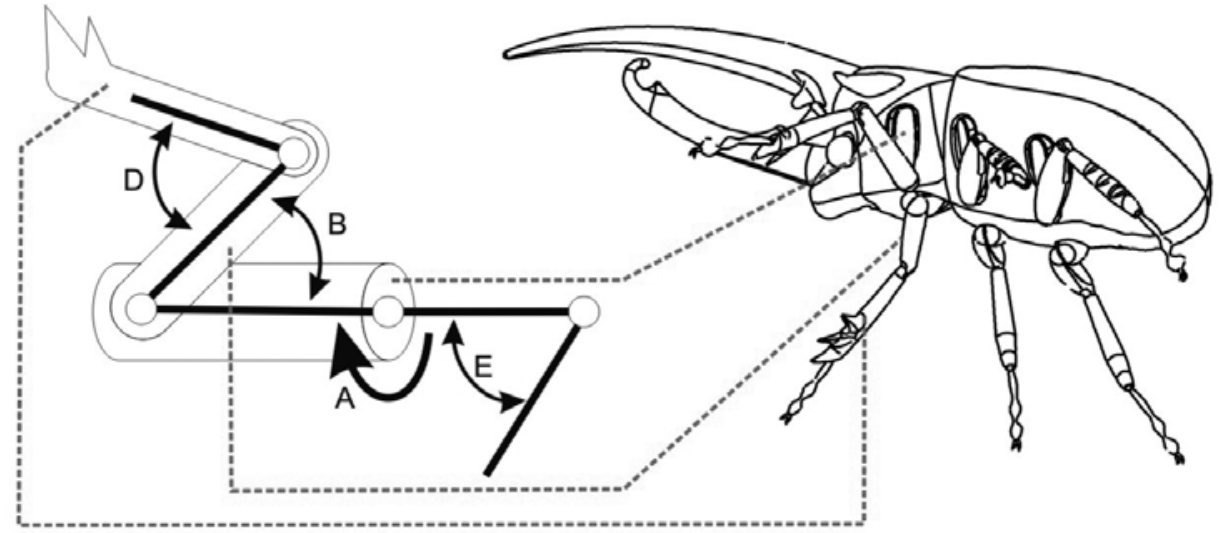

Figura 2: Observación del Dynastes hercules, análisis de los componentes funcionales de sus patas, esquematización de segmentos y articulaciones.os ángulos $A, B, D, E$ son medidos y tabulados en la figura siguiente.

\begin{tabular}{|c|c|c|c|c|c|}
\hline & & \multicolumn{4}{|c|}{ Selección del Miembro (Angulo barrido de movimiento relativo) } \\
\hline Articulación/Junta & Tipo de Junta & Anterior & Medio Anterior & Medio Posterior & Posterior \\
\hline A & 3 & $90^{\circ}$ & $90^{\circ}$ & $90^{\circ}$ & $90^{\circ}$ \\
\hline B & 11 & $45^{\circ}$ & $5^{\circ}$ & $5^{\circ}$ & $50^{\circ}$ \\
\hline D & 11 & $80^{\circ}$ & $70^{\circ}$ & $70^{\circ}$ & $90^{\circ}$ \\
\hline E & 7 & $0^{\circ}$ & $10^{\circ}$ & $10^{\circ}$ & $0^{\circ}$ \\
\hline
\end{tabular}

ESQUEMA DE JUNTAS: 1. Rotación 2. Prismática 3. Helicoidal 4. Cilíndrica 5. Esférica 6. En plano 7. Pasador completa para rotación (con cierre de forma) 8. Corredera completa para traslación (con cierre de forma) 9. Eslabón apoyado contra un plano (con cierre de fuerza) 10. Pasador en ranura (con cierre de forma) 11. Pasador de Primer orden con un GDL (dos eslabones conectados) 12. Pasador de segundo orden con dos GDL (dos eslabones conectados) 13. Rodamiento puro,

Figura 3: Medición de los ángulos de movimiento de las patas del Dynastes hercules. La tabla presenta de modo comparativo los diversos ángulos de movimiento y usa una clasificación convencional de la ingeniería para los esquemas de juntas según diversos tipos de relaciones y movimientos.

Se procede a hacer filmación del animal estudiado, se observan sus movimientos y se establecen ciclos o secuencias de movimientos cuando estas resultan evidentes. Para el caso, el Dynastes hercules presenta en ciertos momentos del movimiento un ciclo de apoyos alternados mediante triángulos opuestos, apoyando de manera alterna la pata frontal y posterior derecha junto con la pata media izquierda (formando un trípode), y alternando este apoyo con el conjunto de la pata frontal y posterior izquierda, junto con la pata media derecha (formando el segundo trípode). 


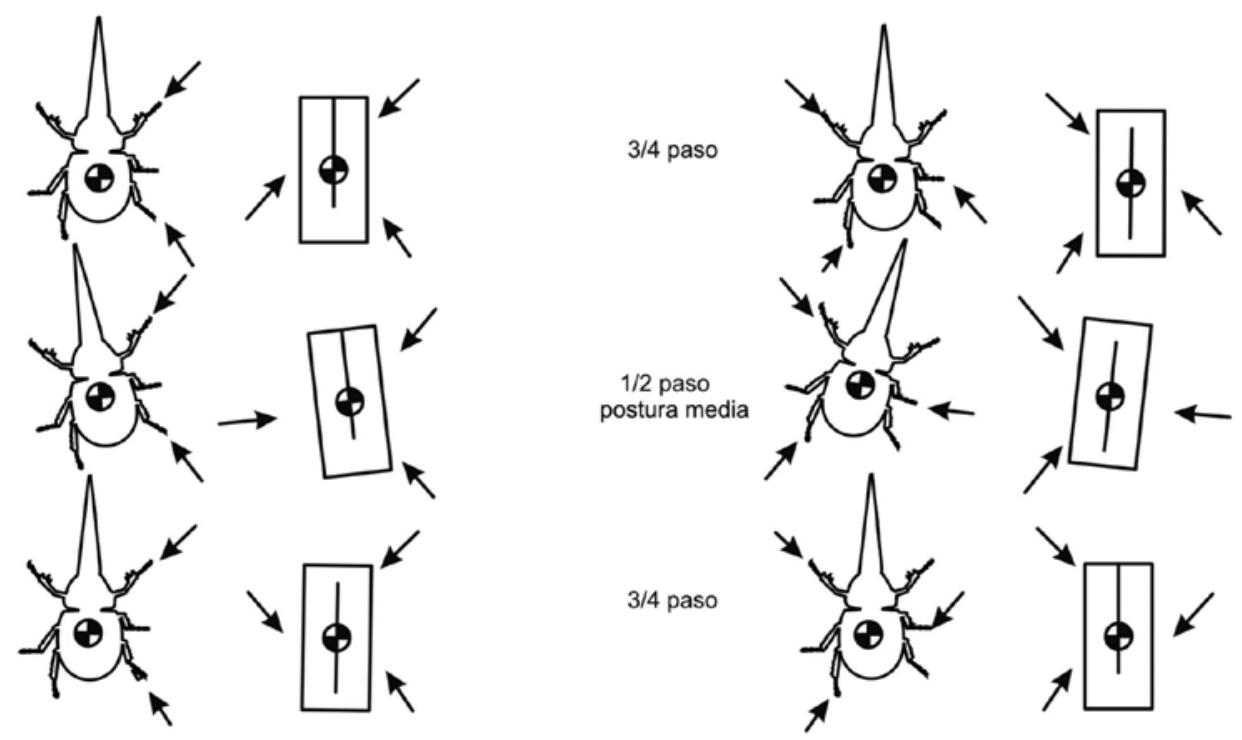

Figura. 3. "representación de ciclos según estados de apoyo". Representación de la secuencia de caminata del escarabajo Dynastes hercules en un ciclo completo. e destaca en esta gráfica que la secuencia se divide en cuartos y cada cuarto corresponde a un momento de estabilidad de la caminata al representar un trípode de apoyo. También presenta como dato importante para el análisis (coincidiendo con Shultz) la posición del cuerpo o su desviación angular respecto a la dirección de avance. Basado en la gráfica y estudio para la cucaracha:The Role of the Mechanical System in Control: A Hypothesis of Self-Stabilization in Hexapedal Runners. Author(s): T. M. Kubow and R. J. FullSource:

Philosophical Transactions: Biological Sciences, Vol. 354, No. 1385, Mechanisms of Neuromoscular Control

(May 29, 1999), pp. 849-86.

Se inicia un estudio detallado del movimiento para comprender la dinámica de la posición del cuerpo del escarabajo respecto a sus momentos de apoyo y movimiento. Este estudio es ante todo cualitativo y no representa realmente el movimiento general de esta especie, se trata de analizar de manera intuitiva y práctica las fuerzas asociadas al movimiento animal.

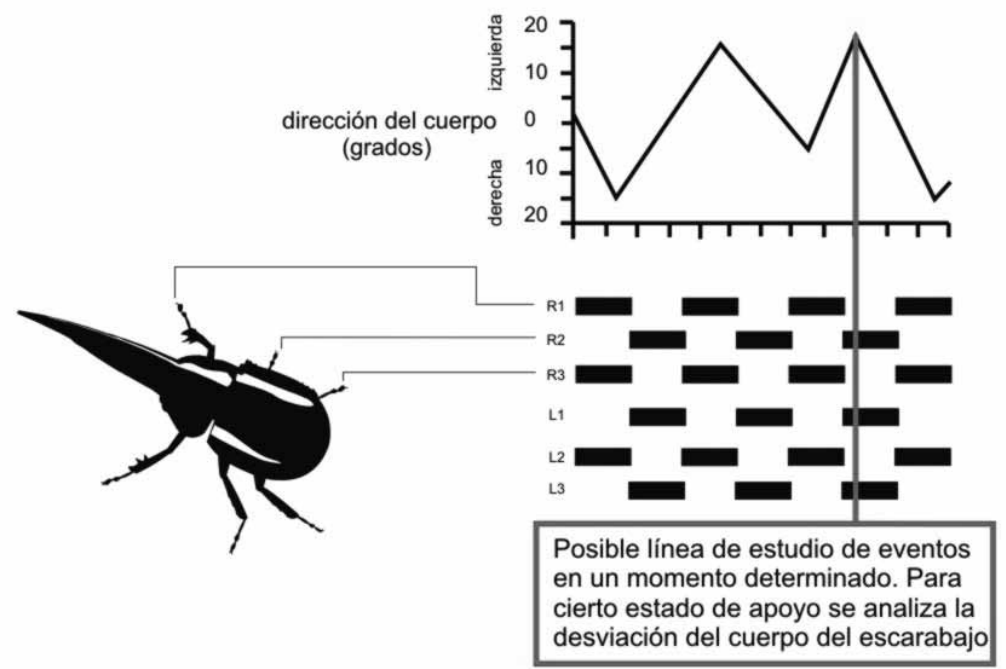

Figura. 4. "Gráficas sincronizadas de variables”. Análisis de la caminata de un escarabajo Dynastes hercules: Se aprecia la nomenclatura de identificación de las patas, el análisis de los tiempos y ciclos de poyo de cada una de las patas y las desviaciones del ángulo de posición del cuerpo del escarabajo con respecto a la dirección de recorrido. Basado en:Walking and surface film locomotion in terrestrial and semi-aquatic spiders by JeffreyW. Shultz. Department of Zoological and Biomedical Sciences, Ohio University, Athens, OH 45701, USA. A la izquierda: Esquema simplificado de la anatomía de la araña y relación gráfica de las patas derechas de la araña con la nomenclatura designada para ellas. .Abajo: anotación sobre la sincronización de "eventos"(apoyos y desviación) en un momento determinado. 


\section{Resultado: diseño de la pata articulada}

Una vez analizados los movimientos, se decide reproducir el funcionamiento de estas estructuras naturales mediante un mecanismo que las reproduzca. El mecanismo propuesto simula este movimiento limitándose al plano de avance del animal, es decir, todos los movimientos que se obtienen suceden en un solo plano coincidente con un plano sagital.

El diseño de la pata articulada se desarrolla tratando de lograr la complejidad del movimiento, y se explica a continuación con dos objetivos: 1. registrar la manera en que metodológicamente fue posible diseñar un mecanismo relativamente complejo como es este, y, 2. ayudar a comprender su funcionamiento. Se entiende el movimiento de la pierna como la sumatoria coordinada de dos movimientos, la flexión y extensión de los elementos y un giro (en un punto análogo a una cadera) que permitiría el avance del mecanismo. Es importante aclarar que estos movimientos deben estar coordinados y que el objetivo de diseño es poder lograr esta coordinación de elementos y movimientos de la forma más sencilla posible. Se trata en este caso de un solo elemento actuador, es decir que no sería aceptable para el diseño tener que proponer actuadores en cada articulación o grupos de actuadores para la extensión y para la rotación.

La primera etapa en el desarrollo del mecanismo fue la reproducción de un mecanismo de flexión y extensión de la pierna. Se observa en la gráfica siguiente un mecanismo de barras paralelas sujeto a un soporte, el diseño hasta acá permite que mediante un solo movimiento todas las articulaciones que conformarán la pierna actúen de manera conjunta, retrayéndose y extendiéndose.

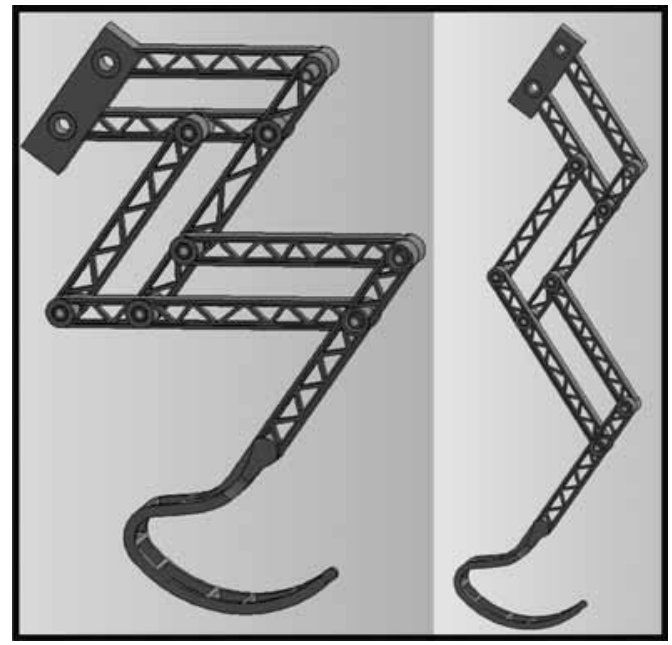

Figura 5. Flexión y extensión: mediante el movimiento de cualquiera de los elementos móviles del mecanismo, todas las piezas desarrollan el movimiento de flexión y extensión.

Se adiciona a este mecanismo de flexión extensión un punto de giro o pivote que da la libertad al mecanismo de producir un giro desde ese punto sin alterar la capacidad de flexión y extensión. 


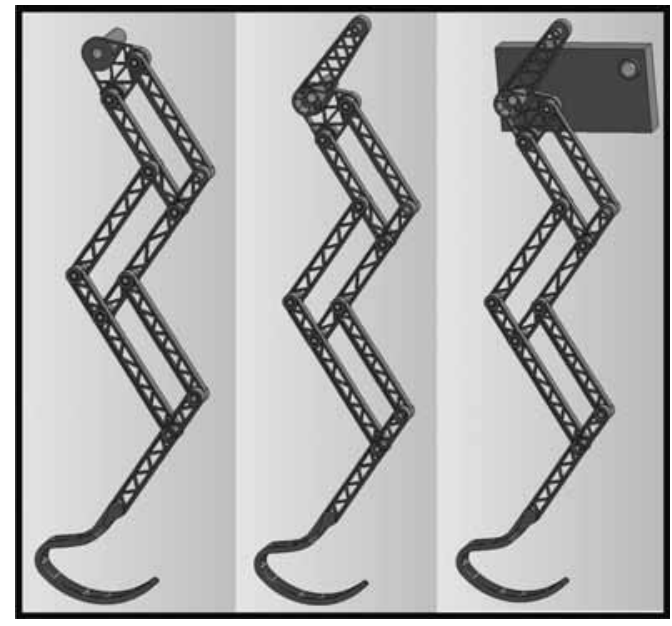

Figura 6. Rotación: Adición de un punto de giro en un punto similar a una "cadera"

A continuación se incluye en el mecanismo un volante que mediante una barra se articula con lo que representaría una "rodilla" en la pierna. El giro de este volante permite lograr el movimiento de flexión y extensión de manera cíclica mediante un movimiento de entrada continuo (de giro).

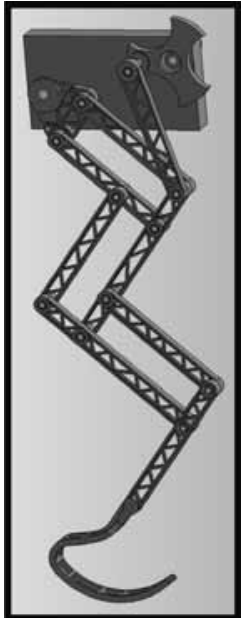

Figura 7. Flexión, extensión y Rotación desvinculada: en este momento el mecanismo puede lograr de manera desvinculada los movimientos de giro y logra una flexión y extensión cíclica.

Un tercer elemento permite vincular los movimientos de flexión y extensión y el movimiento de giro en un ángulo controlado. Queda así simulado el movimiento completo de la pierna articulada.

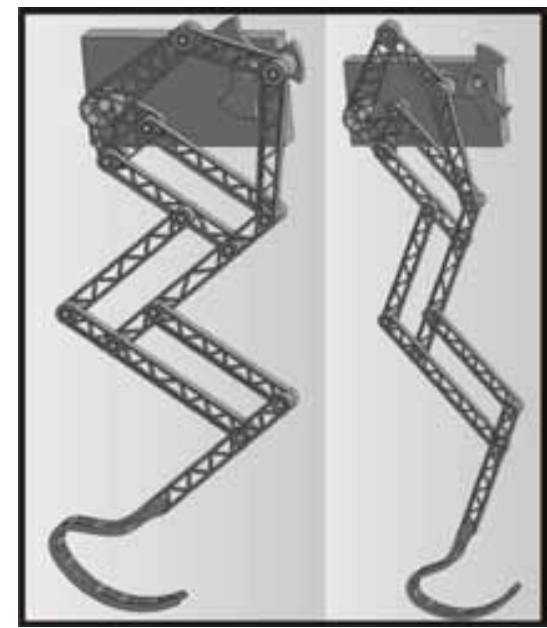

Figura 8. Pierna articulada: mediante un único movimiento de entrada al mecanismo (giro del volante) se logra reproducir el movimiento de una pierna articulada.

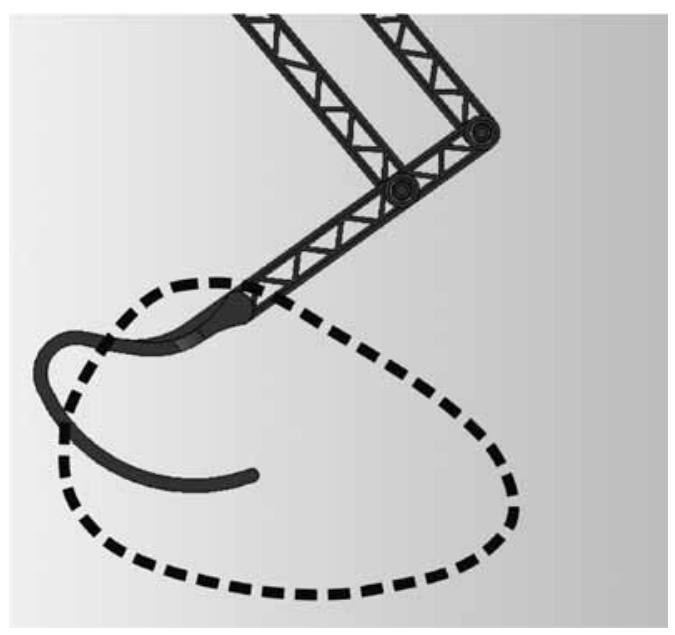

Figura. 9: Ciclograma de movimiento: Representa la manera en que se desplaza en el espacio un punto en la pata de la pierna articulada.

\section{Conclusiones}

Una sola gran conclusión puede ser la apropiada para este proyecto en desarrollo: es viable la construcción de medios alternativos de transporte que permitan alternativas de movilidad sustentables, que se alejen del paradigma del desplazamiento mediante ruedas y que den cabida a soluciones de transporte que no requieran una vasta intervención en el medio para ser adaptados a su funcionamiento. 


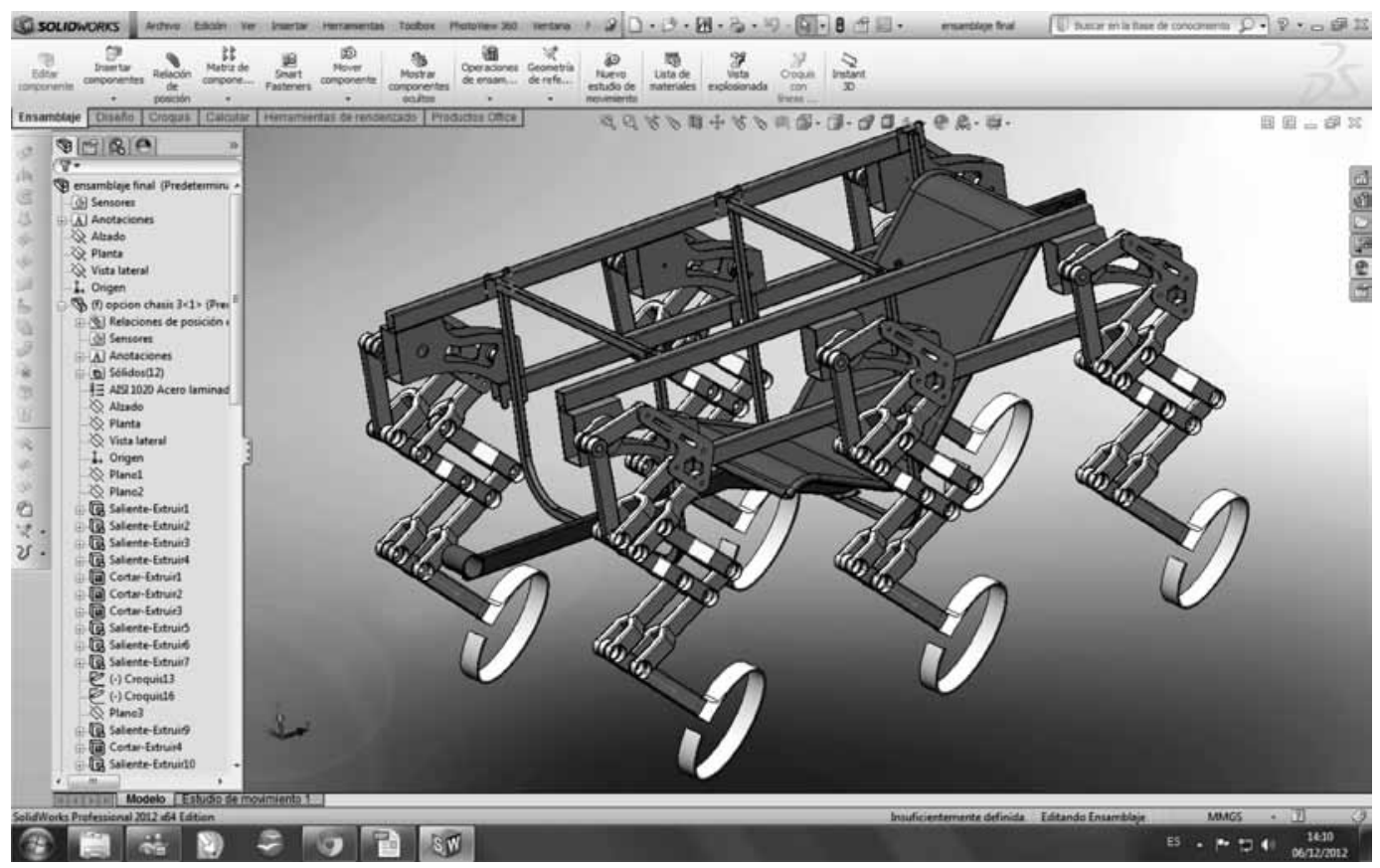

Notas

1. Página web http: / / www.larazon.es/noticia/6907-biomimetica-la-ciencia-extrae-los-secretos-de-la-naturaleza-para-los-grandesinvento (consultado el 20 de abril de 2012 )

\section{Referencias}

Alonso, Carlos \& Nieto, Elena \& Olías Morena, Jesús. (2003). Manual para la Gestión de los Residuos Urbanos. Madrid: La Ley-Actualidad S.A.

Bettini V. (1996). Elementi di Ecologia Urbana. Torino: Einaudi.

Di Pace, M. (2001). Sustentabilidad Urbana y Desarrollo Local, Módulo 4 Curso de Postgrado en Desarrollo Local en Áreas Metropolitanas. San Miguel: Instituto del Conurbano, Universidad Nacional de Gral. Sarmiento.

Kubow \& FullSource. (1999). The Role of the Mechanical System in Control: A Hypothesis of Self-Stabilization in HexapedalRunners, Philosophical Transactions: Biological Sciences. Vol. 354, No. 1385, Mechanisms of Neuromoscular Control.

Lupano, Jorge A. \& Sánchez, Ricardo J. (2009). Políticas de movilidad urbana e infraestructura urbana de transporte. Chile: CEPAL.

Moller, Rolf. (2006). Transporte urbano y desarrollo sostenible en América Latina. Colombia: Programa Editorial Universidad del Valle.

Miralles-Guash \& Cebollada, Ángel. (2003). Movilidad y transporte. Opciones políticas para la ciudad. España: Fundación alternativas.

Ortega y Gasset, José. (1965). Meditación de la técnica. Madrid: Editorial Espasa Calpe.

Velásquez Barrero, Luz Stella. (2004). Propuesta de una metodología de planificación para el desarrollo urbano sostenible y diseño de un sistema de evaluación de la sostenibilidad de ciudades medianas de América Latina. Tesis Doctoral. España: Universidad Politécnica de Catalunya.

Recibido: septiembre 30 / Aprobado: diciembre 1 de 2012 